\title{
CMOS Transistor Mismatch Model valid from Weak to Strong Inversion
}

\author{
Teresa Serrano-Gotarredona and Bernabé Linares-Barranco \\ Instituto de Microelectrónica de Sevilla, Ed. CICA, Av. Reina Mercedes s/n, 41012 Sevilla, SPAIN. \\ Phone: 34-954-5056666, Fax: 34-954-5056686, E-mail: bernabe@imse.cnm.es
}

\begin{abstract}
A five parameter mismatch model continuos from weak to strong inversion is presented. The model is an extension of a previously reported one valid in the strong inversion region [1]. A mismatch characterization of NMOS and PMOS transistors for 30 different geometries has been done with this continuos model. The model is able to predict current mismatch with a mean relative error of $13.5 \%$ in the weak inversion region and 5\% in strong inversion. This is verified for 12 different curves, sweeping $V_{G}, V_{D S}$ and $V_{S}$.
\end{abstract}

\section{Introduction}

Characterization of transistor mismatch is crucial for precision analog design. Using very reduced transistor geometries produces large deviations in the transistor electrical parameters. This may render the analog circuit useless due to unexpected large variations of the circuit specifications. On the contrary, if too conservative transistor geometries are used, the consequence is a waste of area, that also produces an increase in circuit capacitances. This may degrade the speed specifications and increase the circuit power consumption. Thus, a precise mismatch characterization as a function of transistor area is necessary for optimizing area-speed-power consumption in analog design.

Some works on statistical characterization have been previously published in literature [1]-[3]. These works do statistical characterization in the ohmic and/or saturation for the strong inversion region of operation. However, as low-power and low-voltage are becoming increasingly important specifications in analog design, the analog design is moving towards the moderate and weak inversion regions of the transistor operation. Recently, a mismatch model valid for all regions of operation has been published [4]. However, relative errors in the weak inversion region of the order of $100 \%$ are reported. In the present work, we report a continuous 5-parameter mismatch model valid for all regions of operation. The model predicts the current mismatch with a mean relative error of $13.5 \%$ in weak inversion and 5\% in the strong inversion region. The maximum prediction error of our model is less than $50 \%$ for all the operation regions, for 12 different curves. NMOS and PMOS transistors of 30 different geometries have been characterized. The model is an extension of a previously reported 5-parameters mismatch model [1] to the weak inversion region.

\section{Mismatch Model}

To generate a unique mismatch model valid in all regions of operation, a transistor model continuos from weak to strong inversion is necessary [5]-[6]. The present mismatch model is based on the ACM transistor model which is continuos for all regions of operations and is physically based, so that it has a reduced number of physically meaningful parameters [6]. This makes this model especially suitable for transistor mismatch characterization. We have verified that very similar results are obtained if the EKV model [5] is used.

In the ACM model, the current through the transistor $I_{D S}$ is expressed as,

$$
\begin{gathered}
I_{D S}=I_{s}\left(i_{f}\left(V_{p}-V_{S}\right)-i_{r}\left(V_{p}-V_{D}\right)\right) \\
V_{P}-V_{S(D)}=\phi_{t}\left(\sqrt{1+i_{f(r)}}-2+\ln \left(\sqrt{1+i_{f(r)}}-1\right)\right) \\
V_{P}=\frac{V_{G}-V_{T O}}{n} \\
n=1+\frac{\gamma}{2\left(\sqrt{V_{G}-V_{T O}+2 \phi_{F}+\gamma \sqrt{2 \phi_{F}}+1 / 4 \gamma^{2}}-1 / 2 \gamma\right)} \\
I_{S}=I_{S}{ }^{\prime} n=\mu n C^{\prime}{ }_{o x} W / L \phi_{t}^{2} / 2
\end{gathered}
$$

where $V_{G}, V_{S}$ and $V_{D}$ are, respectively, the gate, source and drain voltages referred to the bulk. Parameter $\phi_{t}$ is the thermal voltage; $\gamma$ is the body factor; $\phi_{F}$ is Fermi potential; $\mu$ is mobility; $C_{o x}{ }_{o x}$ is density of oxide capacitance and $W$ and $L$ are the transistor width and length, respectively.

The complete transistor model taking into account some second order effects relevant for mismatch and small transistor geometries, is

$$
I_{D S}=\frac{I_{S}\left(i_{f}-i_{r}\right)\left(1+\lambda\left(V_{D}-V_{S}\right)\right)}{\left(1+\theta_{o}\left[V_{P}-V_{S}\right]^{+}\right)\left(1+\theta_{e} V_{D S_{e f f}}\right)},
$$

where,

$V_{D S_{e f f}}=V_{D S}$ in ohmic region;

$V_{D S_{\text {eff }}}=\left[V_{P}-V_{S}\right]^{+}$in saturation.

Parameter $\lambda$ models the channel pinchoff, and parameters $\theta_{e}$ and $\theta_{o}$ model mobility degradation, velocity saturation and, drain and source series resistances [1]. The operator [ $]^{+}$in equation (2) is a smoothed rectification function as shown in Fig. 1. The function in Fig. 1 is continuos and has continuos derivative. It is defined as, 


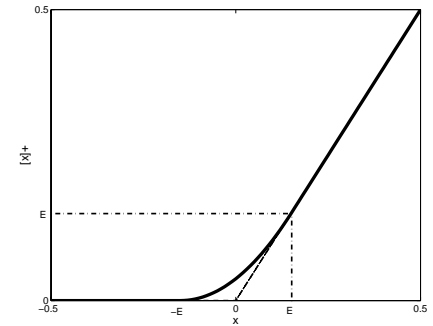

Fig. 1. Smoothed rectification operator

$$
[x]^{+}=\left\{\begin{array}{c}
0 \text { if } x<-E \\
\frac{(x+E)^{2}}{4 E} \text { if }-E<x<E \\
x \text { if } x>E
\end{array}\right.
$$

However, in equation (2), a discontinuity in the derivative still exits in the definition of $V_{D S_{e f f}}$. This problem can be easily solved by expressing $V_{D S_{\text {eff }}}$ as the combination of two smoothed rectification operators,

$$
V_{D S_{e f f}}=\left[V_{p}-V_{S}\right]^{+}-\left[V_{p}-V_{D}\right]^{+} .
$$

The extension of the smoothed region has been empirically chosen to be a fraction of $V_{D S}$, namely, $E=0.3 V_{D S}$.

Our five parameter mismatch model, expresses the current mismatch $\Delta I_{D S} / I_{D S}$ as a first order Taylor series expansion of 5 mismatch parameters $\left\{\Delta I_{S}^{\prime} / I_{s}^{\prime}, \Delta \gamma, \Delta V_{T O}, \Delta \theta_{e}, \Delta \theta_{o}\right\}$,

$$
\begin{gathered}
\frac{\Delta I_{D S}}{I_{D S}}=\frac{\Delta I_{S}^{\prime}}{I_{s}^{\prime}}+\frac{1}{I_{D S}} \frac{\partial I_{D S}}{\partial \gamma} \Delta \gamma+\frac{1}{I_{D S}} \frac{\partial I_{D S}}{\partial V_{T O}} \Delta V_{T O} \\
-\frac{\left[V_{P}-V_{S}\right]^{+}}{1+\theta_{o}\left[V_{P}-V_{S}\right]^{+}} \Delta \theta_{o}-\frac{V_{D S_{e f f}}}{1+\theta_{e} V_{D S_{e f f}}} \Delta \theta_{e}
\end{gathered},
$$

where the set of 5 mismatch parameters $\left\{\Delta I_{S^{\prime}} / I_{s}^{\prime}, \Delta \gamma, \Delta V_{T O}, \Delta \theta_{e}, \Delta \theta_{o}\right\} \quad$ characterizes transistor mismatch for any bias point.

\section{Mismatch Characterization Results}

To characterize the mismatch, arrays of 36 NMOS transistors of 30 different geometries and arrays of 36 PMOS transistors of 30 different geometries were measured accessing to a reduced number of pins [1]. A cell containing 30 different sized NMOS transistors and 30 different sized PMOS transistors is arranged in a $6 \times 6$ matrix.This characterization chip was fabricated in a standard $0.35 \mu \mathrm{m}$ CMOS technology. The 30 geometries correspond to 6 different widths and 5 different transistor lengths.

The transistor widths are: $40 \mu \mathrm{m}, 20 \mu \mathrm{m}, 10 \mu \mathrm{m}, 5 \mu \mathrm{m}$, $2 \mu \mathrm{m}$ and $0.8 \mu \mathrm{m}$.

The transistor lengths are: $10 \mu \mathrm{m}, 5 \mu \mathrm{m}, 2 \mu \mathrm{m}, 0.8 \mu \mathrm{m}$ and $0.35 \mu \mathrm{m}$.

For each transistor in the array, we measured 12 different curves. In each curve, we swept voltage $V_{G}$ while keeping the other voltages constant. The 12 different measured curves correspond to a two dimensional sweep of four $V_{S}$ values and three different $V_{D S}$ voltages. Each curve is measured with 101 data points for $V_{G} \in[0,3.3 \mathrm{~V}]$ and varied in $0.033 \mathrm{~V}$ steps. The
12 measured curves correspond to $V_{S}=\{0 \mathrm{~V}, 0.5 \mathrm{~V}, 1 \mathrm{~V}, 1.5 \mathrm{~V}\}$ and $V_{D S}=\{0.1 \mathrm{~V}, 0.7 \mathrm{~V}, 1.3 \mathrm{~V}\} \cdot V_{G}$ and $V_{S}$ voltages are referred to the local substrate.

To extract the mismatch parameters, first the large signal parameters $\left\{I_{s}^{\prime}, \gamma, \phi_{F}, V_{T O}, \theta_{e}, \theta_{o}, \lambda\right\}$ have to be extracted in order to compute the partial derivatives of equation (5). The large signal parameter extraction is done using nonlinear curve fitting techniques.

To extract the mismatch parameters we compute the current difference $\Delta I$ between two consecutive transistors in the array. This way, we transform the $6 \times 6$ array of transistors into a $5 \times 6$ array of transistor pairs. For each transistor pair, we fit the measured $\Delta I / I$ data for 9 of the curves $\left(V_{S}=\{0 \mathrm{~V}, 0.5 \mathrm{~V}, 1 \mathrm{~V}\}\right.$ and $\left.V_{D S}=\{0.1 \mathrm{~V}, 0.7 \mathrm{~V}, 1.3 \mathrm{~V}\}\right)$ to equation (5). From this fitting, we extract a unique set of 5 mismatch parameters $\left\{\Delta I_{S}{ }^{\prime} / I_{s}{ }^{\prime}, \Delta \gamma, \Delta V_{T O}, \Delta \theta_{e}, \Delta \theta_{o}\right\}$ for each transistor pair. Note that we have not used the 3 curves with $V_{S}=1.5 \mathrm{~V}$ during the extraction of the mismatch parameters. We have left these curves only for evaluation purposes.

For each transistor type (NMOS or PMOS) and for each transistor size, we compute the five standard deviations $\left\{\sigma\left(\Delta I_{s}^{\prime} / I_{s}^{\prime}\right), \sigma(\Delta \gamma), \sigma\left(\Delta V_{T O}\right), \sigma\left(\Delta \theta_{e}\right), \sigma\left(\Delta \theta_{o}\right)\right\}$ and the 10 corresponding correlation terms.

The current mismatch can be predicted using the mismatch parameters, through the theoretical equation,

$$
\begin{gathered}
\sigma^{2}\left(\frac{\Delta I}{I}\right)=\sigma^{2}\left(\frac{\Delta I_{s}^{\prime}}{I_{S^{\prime}}}\right)+\left(\frac{1}{I} \frac{\partial I}{\partial \gamma}\right)^{2} \sigma^{2}(\Delta \gamma)+\left(\frac{1}{I} \frac{\partial I}{\partial V_{T O}}\right)^{2} \sigma^{2}\left(\Delta V_{T O}\right) \\
\left(\frac{1}{I} \frac{\partial I}{\partial \theta_{o}}\right)^{2} \sigma^{2}\left(\Delta \theta_{o}\right)+\left(\frac{1}{I} \frac{\partial I}{\partial \theta_{e}}\right)^{2} \sigma^{2}\left(\Delta \theta_{e}\right)+\text { correlation terms }
\end{gathered}
$$

Fig. 2 shows a comparison, for the 30 geometries of NMOS transistors, between the measured current mismatch (circles) and the current standard deviation computed using the extracted mismatch parameters and equation (6) (solid lines). Fig. 2(a) corresponds to the random current standard deviations measured and computed for $V_{S}=0.5 \mathrm{~V}, V_{D S}=0.1 \mathrm{~V}$ while sweeping the gate voltage $V_{G}$. Fig. 2(a) depicts 6 subfigures, one for each transistor witdh. Each subfigure plots 5 curves, each one corresponding to a different transistor length. Fig. 2(b) corresponds to the random current standard deviations measured and computed for $V_{S}=0.5 \mathrm{~V}$, $V_{D S}=1.3 \mathrm{~V}$ while sweeping the gate voltage $V_{G}$.

In Fig. 3, we show the error between measured and predicted values (in \%) for all 12 curves for NMOS transistors. In each subfigure, the errors are superimposed for all sizes. The mean relative error is $8 \%$ in the weak inversion region and $4 \%$ in strong inversion.

Fig. 4 shows the errors (in \%) between predicted and measured current mismatch. In each subfigure, the errors obtained for the 30 PMOS transistor geometries are superimposed. The mean relative error, in this case, is $13.5 \%$ in the weak inversion region and $5 \%$ in strong inversion. The maximum prediction error of the current mismatch is less than $40 \%$ in the weak inversion region and below $20 \%$ in the strong inversion region.

The current mismatch in Fig. 2, Fig. 3 and Fig. 4 is computed using the five standard deviations $\left\{\sigma\left(\Delta I_{s} / I_{s}\right), \sigma(\Delta \gamma), \sigma\left(\Delta V_{T O}\right), \sigma\left(\Delta \theta_{e}\right), \sigma\left(\Delta \theta_{o}\right)\right\}$ and the 10 possible correlation terms. However, only the three 

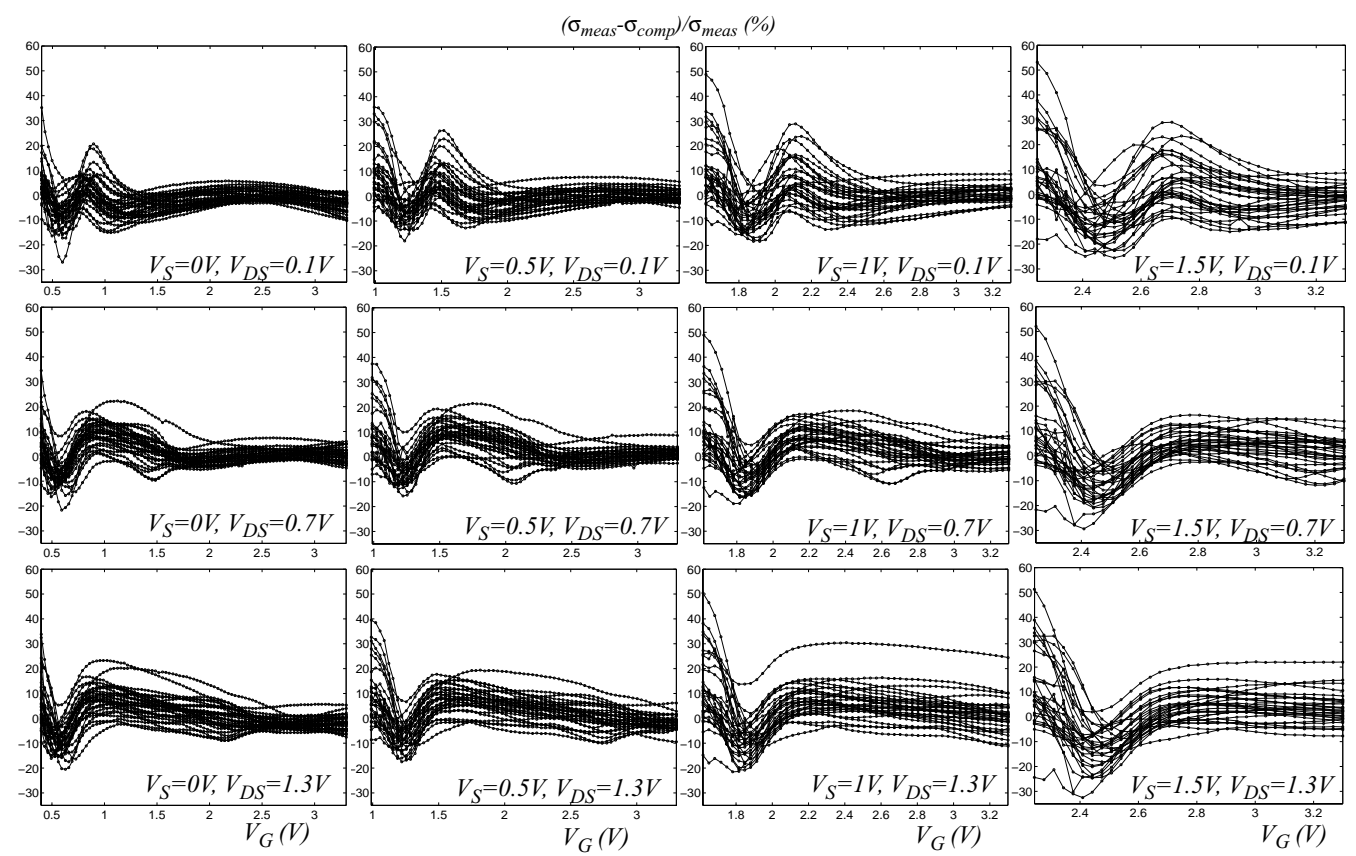

Fig. 3. Errors (in \%) between the measured and computed current random standard deviation for the 30 geometries of NMOS transistors. Each subfigure corresponds to one of the 12 curves $V_{S}=\{0 \mathrm{~V}, 0.5 \mathrm{~V}, 1 \mathrm{~V}, 1.5 \mathrm{~V}\}$ and $V_{D S}=\{0.1 \mathrm{~V}, 0.7 \mathrm{~V}, 1.3 \mathrm{~V}\}$.
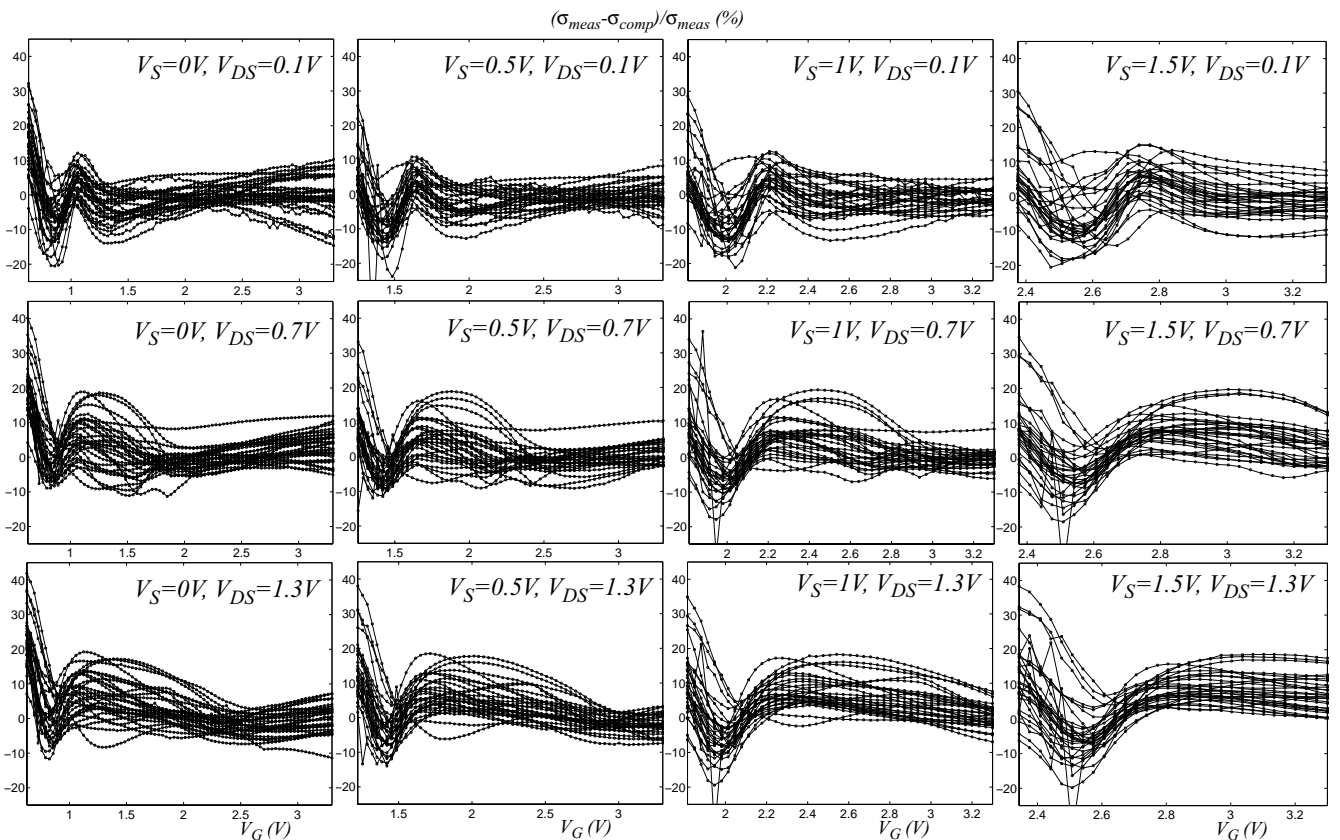

Fig. 4. Errors (in \%) between the measured and computed current random standard deviation for the 30 geometries of PMOS transistors. Each subfigure corresponds to one of the 12 curves $V_{S}=\{0 \mathrm{~V}, 0.5 \mathrm{~V}, 1 \mathrm{~V}, 1.5 \mathrm{~V}\}$ and $V_{D S}=\{0.1 \mathrm{~V}, 0.7 \mathrm{~V}, 1.3 \mathrm{~V}\}$.

correlations $r\left(\Delta I_{s} / I_{s}, \Delta \theta_{e}\right), r\left(\Delta I_{s} / I_{s}, \Delta \theta_{o}\right)$ and $r\left(\Delta \theta_{e}, \Delta \theta_{o}\right)$ are relevant for the NMOS transistor mismatch. For the PMOS transistors we find correlation $r\left(\Delta \gamma, \Delta V_{T O}\right)$ is also important. Fig. 5 depicts the mismatch parameters $\sigma\left(\Delta I_{s} / I_{s}\right), \sigma(\Delta \gamma), \sigma\left(\Delta V_{T O}\right), \sigma\left(\Delta \theta_{e}\right), \sigma\left(\Delta \theta_{o}\right)$ obtained for the NMOS transistors of the 30 different geometries. In Fig. 6 , we draw the mismatch parameters
$\sigma\left(\Delta I_{s} / I_{s}\right), \sigma(\Delta \gamma), \sigma\left(\Delta V_{T O}\right), \sigma\left(\Delta \theta_{e}\right), \sigma\left(\Delta \theta_{o}\right)$ for the PMOS transistors of the 30 different geometries.

\section{Conclusions}

This paper presents a 5 parameter mismatch model valid for all regions of operation. The model is based on a transistor model continous from weak to strong inversion [5]-[6] and a previously reported mismatch model for the 
strong inversion region. This is, to our knowlegde, the first mismatch model published in literature able to predict the current mismatch with mean error less than $13.5 \%$ in all the transistor operation regions, and for such a wide range of transistor curves and geometries.

\section{References}

[1]T. Serrano-Gotarredona and B. Linares-Barranco, "Systematic Width-and Length Dependent CMOS Transistor Mismatch Characterization and Simulation," Analog Integrated Circuits and Signal Processing, vol 21, pp. 271-296, Kluwer Academic Publishers, 1999.

[2]M. J. M. Pelgrom, A. C. J. Duinmaijer, and A. P. G. Welbers, "Matching Properties of MOS Transistors," IEEE Journal of Solid State Circuits, vol. 24, No. 5, pp. 1433-1440, 1989.

[3]J. Bastos, Characterization of MOS Transistor Mismatch for Analog Design, Ph. D. Dissertation, Katholieke Universiteit Leuven, 1998.

[4]J. Croon, M. Rosmeulen, S. Decoutere, and W. Sansen, "An Easy-to-Use Mismatch Model for the MOS Transistor," IEEE Journal of Solid State Circuits, vol. 37, No. 8, pp. 1056-1064, August, 2002.

[5]C.C. Enz, F. Krummernacher and E. A. Vittoz, "An Analytical MOS Transistor Model Valid for All
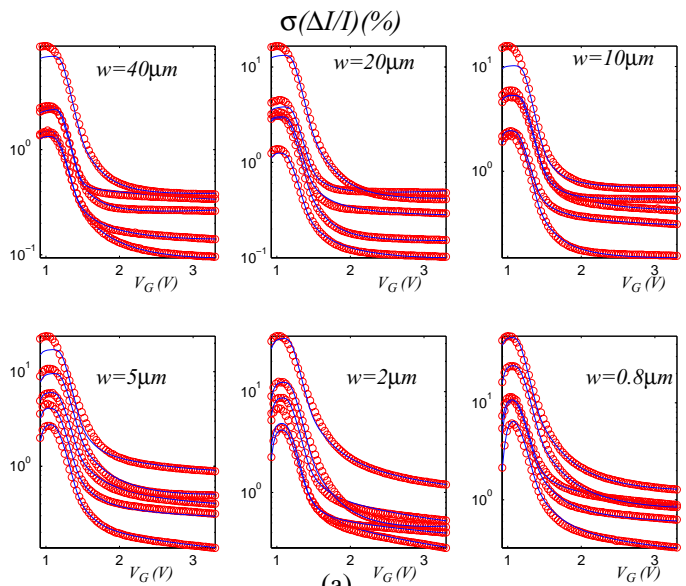

(a)
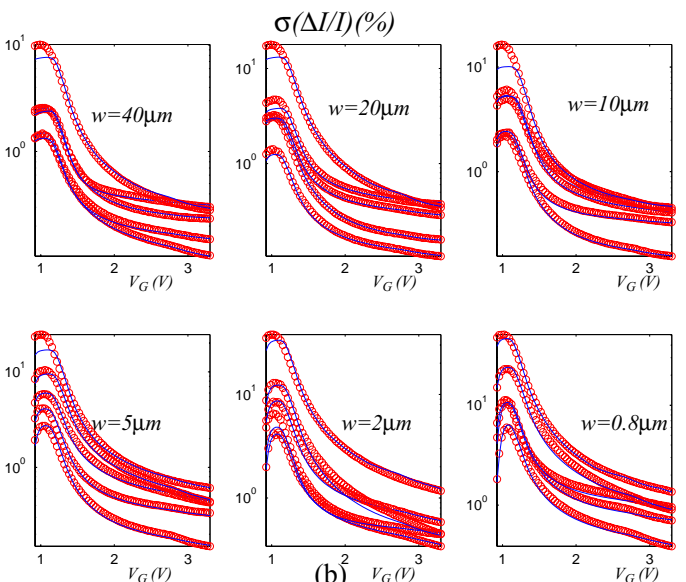

Fig. 2. Comparison between the measured and computed current random standard deviation for the 30 different sizes of NMOS transistors. (a) Curve $V_{s}=0.5 \mathrm{~V}$, $V_{D S}=0.1 \mathrm{~V}$, and (b) curve $V_{S}=0.5 \mathrm{~V}, V_{D S}=1.3 \mathrm{~V}$
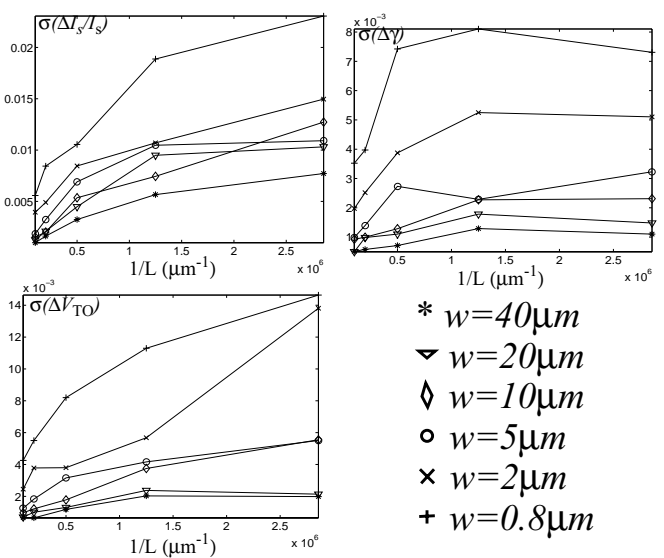

$* w=40 \mu m$

$\nabla w=20 \mu m$

$\Delta w=10 \mu m$

- $w=5 \mu m$

$\times w=2 \mu m$

$+w=0.8 \mu m$
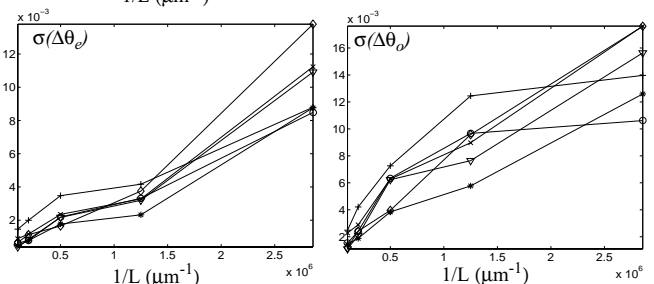

Fig. 6. Mismatch parameters extracted for all the geometries of PMOS transistors

Regions of Operation and Dedicated to Low-Voltage Low-Current Applications," Analog Integrated Circuits and Signal Processing Journal, vol. 8, pp 83-114, July 1995.

[6]C. Galup-Montoro, M. C. Schneider and A. I. A. Cunha, "A Current-Based MOSFET Model for Integrated Circuit Design", Chapter 2 in Low-Voltage/Low-Power Integrated Circuits and Systems, edited by E. Sanchez-Sinencio and A. G. Andreou, IEEE Press, August 1998.
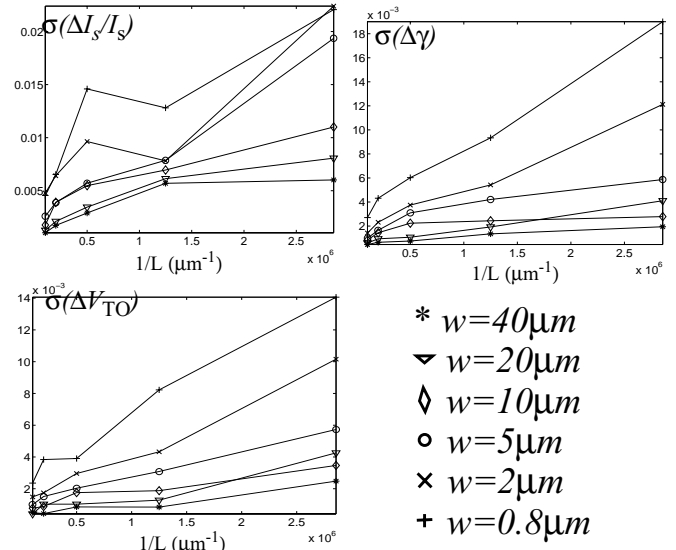

* $w=40 \mu m$

$\nabla w=20 \mu m$

$\diamond w=10 \mu m$

- $w=5 \mu m$

$\times w=2 \mu m$

$+w=0.8 \mu m$
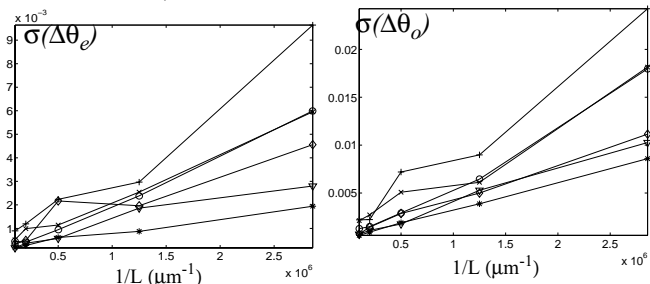

Fig. 5. Mismatch parameters for all the geometries of NMOS transistors 